\title{
ECOLOGICAL STUDIES ON RODENTS IN THE FARM OF AL-AZHAR UNIVERSITY AT ASSIUT GOVERNORATE \\ Abdel-gawad, K.H.*; A.M.Metwally ${ }^{\star *}$;A.M.Nashaat ${ }^{\star * *}$ and A.S. Saudi ** \\ * Faculty of Agriculture Assiut University . \\ ** Faculty of Agriculture Al-Azhar University Cairo. \\ ${ }^{\star * \star}$ Faculty of Agriculture Al-Azhar University Assiut branch
}

\begin{abstract}
Survey of rodents was conducted, at Al-Azhar University Experimental Farm in Assiut. This studies were achieved throughout two year in field crops and woodlands areas. In both areas 50 wire-box traps of the usual spring door $(25 \times 12 \times 10 \mathrm{~cm})$ were baited and distributed twice every 15 days. the Survey of rodents in field crops and woodlands areas revealed the presence of four species of rats and mice. These species were ranked in the field crops as percentage of the total rodents in each year in the following orders, Arvicanthis niloticus (Desm.) (63.09 and 72.08\%), Rattus. Rattus frugivorus (5.58 and 3.6\%), Rattus. r. alexandrinus (1.28 and $5.4 \%$ ) while Mus musculus Linn. ( 30.05 and $18.92 \%$ ) for the first and second year respectively. While in woodlands area were $(37.33 \& 32.8 \%), .(9.22 \& 13.6 \%),(23.05 \&$ $26.4 \%)$ and $(29.95 \& 27.2 \%)$ for the same species and two years respectively. Also, it was found five individuals of the spiny mouse, Acomys cahirinus cahirinus were captured during the same period. Generally, the number of rodents trapped from the field crops and woodlands areas during 2004/2005 (236 individuals) was decreased as compared with the number trapped from the same areas through 2003/2004,( 450 individuals). The decreased of the density may be due to the shortage of food in this area through the last year. In the woodlands area the highest seasonal index was recorded in spring while the lowest one in winter.
\end{abstract}

\section{INTRODUCTION}

In Egypt, rodents problem increased in the last two decades. The main reasons are due to the diversion in agricultural system, land reclamation and construction of new cities in the desert areas, the wide usage of pesticides for controlling agricultural pests lead to rodent natural enemies reduction such as some reptiles and wild birds. Ibrahim (1995) studied the population dynamics of rodent species in certain habitats and found that, the relative abundance of rodent species differed considerably according to the type of habitat and season. The highest population density of rodents was recorded during the spring, following winter crop harvesting, while the lowest was during the winter season. Rattus rattus(Linn.) was abundant in field crop habitats during the spring, summer and autumn seasons, and nearly absent during the winter Sicard et al., (1996) mentioned that 
seasonal changes in reproduction and mobility of tropical rodents depend almost on the amount of food available .

\section{MATERIALS AND METHODS}

Survey of rodents was conducted at Al-Azhar University Experimental Farm in Assiut. This studies were achieved throughout two years from Dec., 2003 to Nov., 2005. It was chosen to resemble the traditional cultivated lands in Upper Egypt. These areas were chosen districts contained many hundred of feddans cultivated with varied field crops, vegetables and fruit trees, and also some sheep farms. There were also many of irrigation, drainage canals, and woodlands.

In both areas 50 wire-box traps of the usual spring door $(25 \times 12 \times 10 \mathrm{~cm})$ were baited and distributed twice every 15 days at 6 $\mathrm{pm}$. with cucumber slices. Next morning, traps were checked, recorded as trap index (No. of rats / trap). The captured rodents were classified into species and subspecies in each locality .

To estimated the population density of rodent species under the field conditions. The capture rodents were classified into species and subspecies. The percentage of every species was estimated monthly and seasonally along the year of the study as trap index. In this study three laboratories were chosen. In every one bait station containing $250 \mathrm{~g}$ from wheat was put, the decrease of the wheat was estimated weekly.

\section{RESULTS AND DISCUSSION}

\section{Survey of rodents:}

The study revealed the occurrence of four rodent species in the field crops :[viz., Arvicanthis niloticus, Mus musculus, Rattus rattus frugivorus, Rattus rattus alexandrinus and Acomys cahirinus (Desm.)]. while In woodlands (viz., A. niloticus, $M$. musculus, $R$. r. frugivorus and Rattus rattus alexandrinus) (Tables 1 and 2).

\section{1-In field crops :}

The abundance of rodent species in the first and second year could be arranged quantitatively in the following descending order: The Nile grass rat, $A$. niloticus $(63.09$ and $72.07 \%)$. The House mouse, $M$. musculus (30.04 and $18.92 \%$ ) The White bellied rat, $R$. r. frugivorus (5.58 and 3.6\%). The Gray bellied rat, $R$. r. alexandrinus (1.29 and $5.41 \%)$ for the first and second year, respectively. and five individuals of Spiny mouse, A. c. cahirinus were recorded throughout the study years. similar results obtained by Abdel-Gawad et al., (1982), Abazaid (1990) and Abdel-Galil (2005) they found different between species through the year at same field. 
Abdel-gawad, K.H. et al.

1

8189 
J. Agric. Sci. Mansoura Univ., 33(11), November, 2008

2

8190 


\section{2- In the woodlands area :}

The Nile grass rat was the most dominant species constituting $37.33 \%$ and $32.8 \%$ of the total trapped rodents for both first and second year. Followed by the house mouse $29.95 \%$ and $27.2 \%$. then the gray bellied rat $23.5 \%$ and $26.4 \%$. and the white bellied rat was the last species of occurrence $9.22 \%$ and $13.6 \%$ for the first and second year respectively. Concerning the percentage of the total numbers of the trapped rodents during $2003 / 2004$ in the two areas of study were $51.77 \%$ in the field crops and $48.23 \%$ in the woodland area , while 2004/2005.were (47.04\%) in the field crops and (52.96 $\%)$ in the woodland area. The high population density of rodents in the both areas may be due mainly to the availability of food and shelters in these areas. On the basis of density percentages, $A$. niloticus ranked the first constituting $63.09 \% \& 37.33 \%$ of the total trapped rodents from the field crops and woodland areas during the first year.

While, $72.07 \% \& 32.8 \%$ of the total rodent trapped from the same areas in the second year, respectively. It may be concluded that the availability of preferred food in both areas led the rats to feed, and produce higher reproduction rat in both areas, wholly in agreement with the findings obtained by Abdel-Gawad et al., (1987) and El-Feky (1990). Khalid et al. (1992) Mourad (1997) they found the density of rodents depend on availability of food and $A$. niloticus was the major one.

\section{Population density of rodents \\ 1- in the field crops:}

Data in (Table 3 and 4) show that the highest seasonal index of rodent during 2003/2004 was recorded in winter (0.27) while the lowest one in autumn (0.113) the peak of rodent population was recorded in January (0.43) and the least density was recorded in December (0.07). This may be due to the stay of the rodent in their shelters to avoid the cold weather during December and go out their shelters to bring their food during January. On the other hand, in $2004 / 2005$ the highest seasonal indices of rodents were observed in Summer (0.123) and the lowest in spring (0.07). the peak of rodent population in 2004/2005 was recorded in July (0.14) and the least density during April (0.05). The same results founded by Abdel-Gawad et al., (1982) Zaghloul and Zakaria (1986) and El-Deeb et al. (1992b) they found the population density of rodents differed from season to another

\section{2- in the woodlands:}

As shown in (Tables 5 and 6 ) the highest rodents seasonal indices of during 2003/2004 was obtained in spring (0.350) and the lowest in winter $(0.073)$. In March the density of rodent population reached its maximum (0.39), whereas the least density reached its minimum in February (0.05). The highest seasonal indices of rodents during 2004/2005 was in summer (0.147) and the lowest in winter (0.073). 
J. Agric. Sci. Mansoura Univ., 33(11), November, 2008 
Abdel-gawad, K.H. et al.

8193 
J. Agric. Sci. Mansoura Univ., 33(11), November, 2008

5

8194 
Abdel-gawad, K.H. et al.

6

8195 
In May the density of rodent population reached its maximum (0.19), whereas the least density reached its minimum in April (0.03). The decrease of the density through April may be due to the migratory of rodent from the woodlands to the wheat fields or to the storage. This data was in agreement with the obtained by Maher Ali et.al.(1974) ;Abdel Gawad (1979). Ali, M.K. (1985); Yossef, A.E. (1996) ; Embarak, M.Z. (1997) and Ahmed, M.Y.M. (2001) they found that the population density of the rats mains on food and climatic factors .

\section{REFERENCES}

Abazaid, A.A. (1990): Efficiency of some common and used rodenticides and some new alternative against rodents in Qena governorate. M. Sc. Thesis, Fac. Agric., Assiut Univ., pp. 93.

Abdel-Galil, Y. M.A. (2005): Comparative studies on rodenticides against some rodents. Ph. D. Thesis, Fac. Agric., Al-Azhar Univ. Egypt. pp. 158.

Abdel-Gawad, K.H. (1979): Studies on the interrelation between rodents and their ectoparasites in the cultivated and semi-arid zones. Ph. D. Thesis, Fac. Agric., Assiut Univ. $141 \mathrm{pp}$.

Abdel-Gawad, K.H.; Farghal, A.I.; Salman, A.G. and Ali, M.K. (1987): Survey and population density of rodent species in some urban, rural and cultivated areas in Sohag governorate, Upper Egypt. Assiut J. Agric. Sci., 18(1): 259-266.

Abdel-Gawad, K.H.; Salit, A.M. and Maher Ali, A. (1982): Population density of rodent species in agriculture and semi-arid area. Assiut J. Agric. Sci., 13(2): 27-36.

Ahmed, M.Y.M. (2001): Studies on the field rat. M. Sc. Thesis, Fac. Agric., Al-Azhar Univ., pp.144.

Ali, M.K. (1985): Studies on rodents and their ectoparasites in Sohag, governorate. M. Sc. Thesis, Fac. Agric. Assiut Univ. pp. 137.

El-Deeb, H.I.; Lokma, H.E. and El-Fishawi, A.A. (1992b): Field studies on population dynamics and reproductive biology of the Nile rat, Arvicanthis niloticus. Zagazig J. Agric. Res., 9(3): 1431-1435. 10

El-Feky, M.A. (1990): Studies on small rodents and their fleas species of public health importance in Sabahiya Experimental Station with special reference to their control. M. Sc. Thesis, Fac. Agric., Alex. Univ., pp.113.

Embarak, M.Z. (1997): Ecological and control studies on rodents and their ectoparasites in cultivated and newly-reclaimed areas. M. Sc. Thesis, Fac. Agric., Assiut Univ. 130 pp.

Ibrahim, I.K. (1995): Studies on the toxicity effect of some substances on rodents in Egypt. M. Sc. Thesis, Fac. Agric. Assiut Univ. pp. 153.

Khalid, M.L.M.; Morsy, T.A.; El-Shennawy, S.F.A. and Farag, A.M.M.K. (1992): Studies on flea fauna in El-Fayoum governorate, Egypt. Journal of Egyptian Society of Parasitology, 22(3): 783-799. 
Maher Ali, A.; Salit, A.M.; Sheta, I. and Mourad, M.G. (1974): Burrowing rodents in the Nile Valley, upper Egypt. Zool. Soci. Egypt. Bull. 26: 32.

Mourad, A.A.M. (1997): Ecological, Biological and Toxicological studies on rodents' species at the Newly Reclaimed Lands. M. Sc. Thesis, Institute of Environmental studies and Researches, Ain Shams University, Egypt. pp. 190.

Sicard, B.; Papillon, Y. and Fuminier, F. (1996): Trophic and nontrophic factors and life cycle of soudano-sahehen rodents. International Workshop on Rodent Biology and Integrated Pest Management in Africa, Sokoine Univ. Of Agric., Morogoro. Tanzania 21-25 Oct. 1996. P:19.

Yossef, A.E. (1996): Ecological biological and toxicological studies on rats stores and Shoguns. Ph. D. Thesis, Fac. Agric., Menofia Univ., Egypt. pp. 132.

Zaghloul, T.M. and Zakaria, M. (1986): Laboratory evaluation of certain Biocides against the house mouse, Mus musculus L. in recent advances in rodent control. Proceeding of The Second Symposium On Recent Advances In Rodent Control. Kuwait, February, $2^{\text {nd }}: 19-27$.

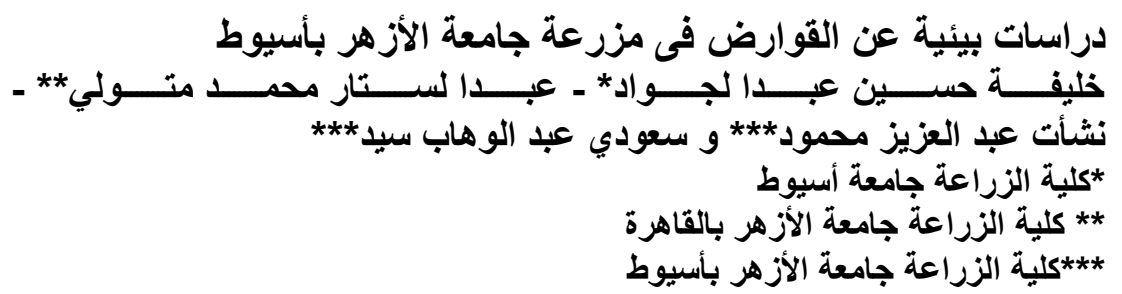

أجريت بعض الدراسات على القوارض فى مزرعة كلية الزراعة جامعة الأزهر بأسيوط

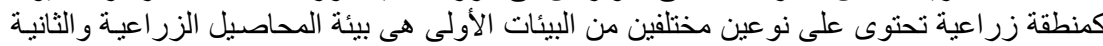

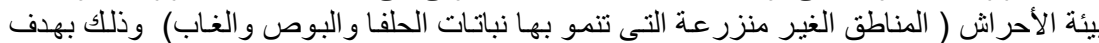

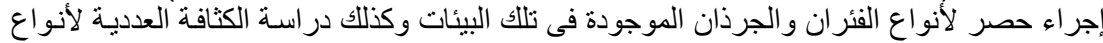

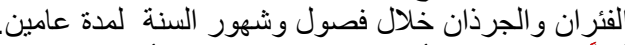

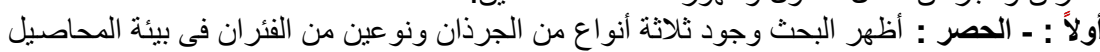

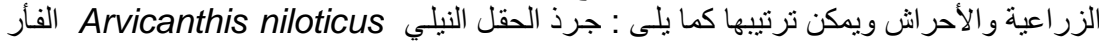

، Rattus rattus frugivorus جرد النخيل ذو البطن البيضاء

الجرذّ المتسلق ذو البطن الرمادي Rattus rattus alexandrinus الفأر الثوكي القاهري . Acomys cahirinus cahirinus

ثُانيا:- التذبذبات الثهرية والموسمية للقوارض : تم دراسة التذبذبات الثهرية و الموسمية لأعداد

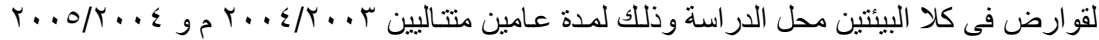

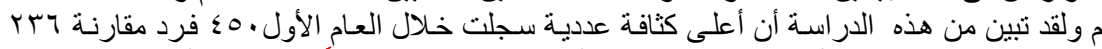

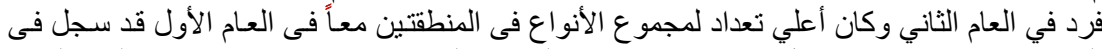

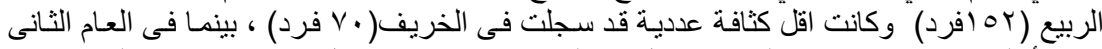

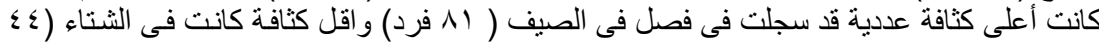


J. Agric. Sci. Mansoura Univ., 33(11), November, 2008

8198 
Abdel-gawad, K.H. et al.

8199 

Table (3): Monthly and seasonal distribution of rodent species in the field crops, at Al-Azhar University Exptl. Farm, Assiut (2003-2004).

\begin{tabular}{|c|c|c|c|c|c|c|c|c|c|c|c|}
\hline \multirow{2}{*}{$\begin{array}{l}\text { Month } \\
\text { Season }\end{array}$} & \multirow[t]{2}{*}{ and } & \multicolumn{2}{|c|}{ Arvicanthus niloticus } & \multicolumn{2}{|c|}{$\begin{array}{c}\text { Rattus rattus } \\
\text { frugivorus }\end{array}$} & \multicolumn{2}{|c|}{$\begin{array}{l}\text { Rattus rattus } \\
\text { alexandrinus }\end{array}$} & \multicolumn{2}{|c|}{ Mus musculus } & \multicolumn{2}{|c|}{ Total rodent spp. } \\
\hline & & $\begin{array}{l}\text { No. } \\
\text { rat }\end{array}$ & Trap index & $\begin{array}{l}\text { No. } \\
\text { rat }\end{array}$ & Trap index & $\begin{array}{l}\text { No. } \\
\text { rat }\end{array}$ & Trap index & No. mice & $\begin{array}{c}\text { Trap } \\
\text { index }\end{array}$ & No. rodent & $\begin{array}{l}\text { Trap } \\
\text { index }\end{array}$ \\
\hline Dec. & & 6 & 0.06 & 0 & 0 & 0 & 0 & 1 & 0.01 & 7 & 0.07 \\
\hline $\begin{array}{l}\text { Jan. } \\
\end{array}$ & & 21 & 0.21 & 9 & 0.09 & 0 & 0 & 13 & 0.13 & 43 & 0.43 \\
\hline Feb. & & 15 & 0.15 & 2 & 0.02 & 0 & 0 & 14 & 0.14 & 31 & 0.31 \\
\hline Winter & & 42 & 0.14 & 11 & 0.036 & 0 & 0 & 28 & 0.093 & 81 & 0.27 \\
\hline Mar. & & 8 & 0.08 & 1 & 0.01 & 0 & 0 & 8 & 0.08 & 17 & 0.17 \\
\hline Apr. & & 7 & 0.07 & 0 & 0 & 0 & 0 & 7 & 0.07 & 14 & 0.14 \\
\hline May & & 10 & 0.10 & 0 & 0 & 0 & 0 & 6 & 0.06 & 16 & 0.16 \\
\hline Spring & & 25 & 0.083 & 1 & 0.003 & 0 & 0 & 21 & 0.07 & 47 & 0.156 \\
\hline Jun. & & 15 & 0.15 & 0 & 0 & 0 & 0 & 8 & 0.08 & 23 & 0.23 \\
\hline Jul. & & 23 & 0.23 & 0 & 0 & 0 & 0 & 4 & 0.04 & 27 & 0.27 \\
\hline Aug. & & 16 & 0.16 & 1 & 0.01 & 1 & 0.01 & 3 & 0.03 & 21 & 0.21 \\
\hline Summer & & 54 & 0.18 & 1 & 0.003 & 1 & 0.003 & 15 & 0.05 & 71 & 0.237 \\
\hline Sep. & & 9 & 0.09 & 0 & 0 & 0 & 0 & 3 & 0.03 & 12 & 0.12 \\
\hline Oct. & & 9 & 0.09 & 0 & 0 & 1 & 0.01 & 2 & 0.02 & 12 & 0.12 \\
\hline Nov. & & 8 & 0.08 & 0 & 0 & 1 & 0.01 & 1 & 0.01 & 10 & 0.10 \\
\hline Autumn & & 26 & 0.086 & 0 & 0 & 2 & 0.007 & 6 & 0.02 & 34 & 0.113 \\
\hline G. total & & 147 & 0.122 & 13 & 0.011 & 3 & 0.003 & 70 & 0.058 & 233 & 0.194 \\
\hline
\end{tabular}


Table (4): Monthly and seasonal distribution of rodent species in the field crops, at Al-Azhar University Exptl. Farm, Assiut (2004-2005).

\begin{tabular}{|c|c|c|c|c|c|c|c|c|c|c|}
\hline \multirow{2}{*}{$\begin{array}{l}\text { Month } \\
\text { Season }\end{array}$} & \multicolumn{2}{|c|}{ Arvicanthus niloticus } & \multicolumn{2}{|c|}{$\begin{array}{c}\text { Rattus rattus } \\
\text { frugivorus }\end{array}$} & \multicolumn{2}{|c|}{$\begin{array}{l}\text { Rattus rattus } \\
\text { alexandrinus }\end{array}$} & \multicolumn{2}{|c|}{ Mus musculus } & \multicolumn{2}{|c|}{ Total rodent spp. } \\
\hline & $\begin{array}{l}\text { No. } \\
\text { Rat }\end{array}$ & Trap index & $\begin{array}{l}\text { No. } \\
\text { rat }\end{array}$ & Trap index & $\begin{array}{l}\text { No. } \\
\text { rat }\end{array}$ & Trap index & No. mice & $\begin{array}{l}\text { Trap } \\
\text { index }\end{array}$ & No. rodent & $\begin{array}{l}\text { Trap } \\
\text { index }\end{array}$ \\
\hline Dec. & 7 & 0.07 & 0 & 0 & 0 & 0 & 1 & 0.010 & 8 & 0.08 \\
\hline Jan. & 5 & 0.05 & 3 & 0.03 & 0 & 0 & 0 & 0 & 8 & 0.08 \\
\hline Feb. & 4 & 0.04 & 1 & 0.01 & 1 & 0.01 & 0 & 0 & 6 & 0.06 \\
\hline Winter & 16 & 0.053 & 4 & 0.013 & 1 & 0.003 & 1 & 0.003 & 22 & 0.073 \\
\hline Mar. & 6 & 0.06 & 0 & 0 & 0 & 0 & 0 & 0 & 6 & 0.06 \\
\hline Apr. & 3 & 0.03 & 0 & 0 & 1 & 0.01 & 1 & 0.010 & 5 & 0.05 \\
\hline May & 8 & 0.08 & 0 & 0 & 0 & 0 & 2 & 0.020 & 10 & 0.1 \\
\hline Spring & 17 & 0.057 & 0 & 0 & 1 & 0.003 & 3 & 0.010 & 21 & 0.07 \\
\hline \begin{tabular}{|l} 
Jun. \\
\end{tabular} & 9 & 0.09 & 0 & 0 & 0 & 0 & 4 & 0.040 & 13 & 0.13 \\
\hline Jul. & 11 & 0.11 & 0 & 0 & 0 & 0 & 3 & 0.030 & 14 & 0.14 \\
\hline Aug. & 9 & 0.09 & 0 & 0 & 0 & 0 & 1 & 0.010 & 10 & 0.1 \\
\hline Summer & 29 & 0.097 & 0 & 0 & 0 & 0 & 8 & 0.027 & 37 & 0.123 \\
\hline Sep. & 5 & 0.05 & 0 & 0 & 2 & 0.02 & 3 & 0.030 & 10 & 0.1 \\
\hline Oct. & 9 & 0.09 & 0 & 0 & 1 & 0.01 & 1 & 0.010 & 11 & 0.11 \\
\hline Nov. & 4 & 0.04 & 0 & 0 & 1 & 0.01 & 5 & 0.050 & 10 & 0.1 \\
\hline Autumn & 18 & 0.06 & 0 & 0 & 4 & 0.013 & 9 & 0.030 & 31 & 0.103 \\
\hline G. total & 80 & 0.067 & 4 & 0.003 & 6 & 0.005 & 21 & 0.018 & 111 & 0.092 \\
\hline
\end{tabular}

Table (5): Monthly and seasonal distribution of rodent species in the woodlands, (2003-2004). 


\begin{tabular}{|c|c|c|c|c|c|c|c|c|c|c|}
\hline \multirow{2}{*}{$\begin{array}{ll}\begin{array}{l}\text { Month } \\
\text { Season }\end{array} & \text { and }\end{array}$} & \multicolumn{2}{|c|}{$\begin{array}{c}\text { Arvicanthus } \\
\text { niloticus }\end{array}$} & \multicolumn{2}{|c|}{$\begin{array}{l}\text { Rattus rattus } \\
\text { Frugivorus }\end{array}$} & \multicolumn{2}{|c|}{$\begin{array}{l}\text { Rattus rattus } \\
\text { Alexandrinus }\end{array}$} & \multicolumn{2}{|c|}{ Mus musculus } & \multicolumn{2}{|c|}{ Total rodent spp. } \\
\hline & $\begin{array}{l}\text { No. } \\
\text { Rat }\end{array}$ & Trap index & $\begin{array}{l}\text { No. } \\
\text { rat }\end{array}$ & Trap index & $\begin{array}{l}\text { No. } \\
\text { rat }\end{array}$ & Trap index & $\begin{array}{l}\text { No. } \\
\text { mice }\end{array}$ & $\begin{array}{l}\text { Trap } \\
\text { index }\end{array}$ & No. rodent & $\begin{array}{l}\text { Trap } \\
\text { index }\end{array}$ \\
\hline Dec. & 2 & 0.02 & 0 & 0 & 6 & 0.06 & 2 & 0.02 & 10 & 0.1 \\
\hline Jan. & 2 & 0.02 & 0 & 0 & 5 & 0.05 & 0 & 0 & 7 & 0.07 \\
\hline Feb. & 2 & 0.02 & 0 & 0 & 2 & 0.02 & 1 & 0.01 & 5 & 0.05 \\
\hline Winter & 6 & 0.02 & 0 & 0 & 13 & 0.04 & 3 & 0.01 & 22 & 0.073 \\
\hline Mar. & 12 & 0.12 & 4 & 0.04 & 10 & 0.1 & 13 & 0.13 & 39 & 0.39 \\
\hline Apr. & 13 & 0.13 & 2 & 0.02 & 9 & 0.09 & 11 & 0.11 & 35 & 0.35 \\
\hline May & 20 & 0.20 & 2 & 0.02 & 1 & 0.01 & 8 & 0.08 & 31 & 0.31 \\
\hline Spring & 45 & 0.15 & 8 & 0.03 & 20 & 0.07 & 32 & 0.1 & 105 & 0.35 \\
\hline Jun. & 5 & 0.05 & 6 & 0.06 & 3 & 0.03 & 10 & 0.1 & 24 & 0.24 \\
\hline \begin{tabular}{|l|} 
Jul. \\
\end{tabular} & 6 & 0.06 & 2 & 0.01 & 2 & 0.02 & 9 & 0.09 & 18 & 0.18 \\
\hline Aug. & 3 & 0.03 & 1 & 0.02 & 2 & 0.02 & 5 & 0.05 & 12 & 0.12 \\
\hline Summer & 14 & 0.05 & 9 & 0.03 & 7 & 0.02 & 24 & 0.08 & 54 & 0.18 \\
\hline Sep. & 7 & 0.07 & 3 & 0.03 & 1 & 0.01 & 2 & 0.02 & 13 & 0.13 \\
\hline Oct. & 5 & 0.05 & 0 & 0 & 3 & 0.03 & 3 & 0.03 & 11 & 0.11 \\
\hline Nov. & 4 & 0.04 & 0 & 0 & 7 & 0.07 & 1 & 0.01 & 12 & 0.12 \\
\hline Autumn & 16 & 0.05 & 3 & 0.01 & 11 & 0.07 & 6 & 0.02 & 36 & 0.12 \\
\hline G. total & 81 & 0.068 & 20 & 0.017 & 51 & 0.043 & 65 & 0.054 & 217 & 0.181 \\
\hline
\end{tabular}

Table (6): Monthly and seasonal distribution of rodent species in the woodlands, (2004-2005).

\begin{tabular}{|l|l|l|l|l|l|}
\hline Month and & Arvicanthus niloticus & Rattus rattus & Rattus rattus & Mus musculus & Total rodent spp. \\
\hline
\end{tabular} 


\section{J. Agric. Sci. Mansoura Univ., 33(11), November, 2008}

\begin{tabular}{|c|c|c|c|c|c|c|c|c|c|c|}
\hline \multirow[t]{2}{*}{ Season } & & & \multicolumn{2}{|c|}{ Frugivorus } & \multicolumn{2}{|c|}{ Alexandrinus } & \multirow[b]{2}{*}{ No. mice } & \multirow[b]{2}{*}{$\begin{array}{l}\text { Trap } \\
\text { index }\end{array}$} & \multirow[b]{2}{*}{ No. rodent } & \multirow[b]{2}{*}{$\begin{array}{c}\text { Trap } \\
\text { index }\end{array}$} \\
\hline & $\begin{array}{l}\text { No. } \\
\text { rat }\end{array}$ & Trap index & $\begin{array}{l}\text { No. } \\
\text { rat }\end{array}$ & Trap index & $\begin{array}{l}\text { No. } \\
\text { rat }\end{array}$ & Trap index & & & & \\
\hline Dec. & 2 & 0.02 & 0 & 0 & 6 & 0.06 & 2 & 0.020 & 10 & 0.1 \\
\hline \begin{tabular}{|l}
$J a n$. \\
\end{tabular} & 2 & 0.02 & 0 & 0 & 5 & 0.05 & 0 & 0 & 7 & 0.07 \\
\hline Feb. & 2 & 0.02 & 0 & 0 & 2 & 0.02 & 1 & 0.010 & 5 & 0.05 \\
\hline Winter & 6 & 0.02 & 0 & 0 & 13 & 0.034 & 3 & 0.010 & 22 & 0.073 \\
\hline Mar. & 5 & 0.05 & 0 & 0 & 4 & 0.04 & 2 & 0.020 & 11 & 0.11 \\
\hline Apr. & 2 & 0.02 & 0 & 0 & 0 & 0 & 1 & 0.010 & 3 & 0.03 \\
\hline May & 9 & 0.09 & 3 & 0.03 & 0 & 0 & 7 & 0.070 & 19 & 0.19 \\
\hline Spring & 16 & 0.053 & 3 & 0.01 & 4 & 0.013 & 10 & 0.033 & 33 & 0.11 \\
\hline Jun. & 4 & 0.04 & 5 & 0.05 & 4 & 0.04 & 4 & 0.040 & 17 & 0.17 \\
\hline Jul. & 5 & 0.05 & 2 & 0.02 & 3 & 0.03 & 5 & 0.050 & 15 & 0.15 \\
\hline Aug. & 1 & 0.01 & 2 & 0.02 & 2 & 0.02 & 7 & 0.070 & 12 & 0.12 \\
\hline Summer & 10 & 0.033 & 9 & 0.03 & 9 & 0.03 & 16 & 0.053 & 44 & 0.147 \\
\hline Sep. & 5 & 0.05 & 0 & 0 & 2 & 0.02 & 2 & 0.020 & 9 & 0.09 \\
\hline Oct. & 1 & 0.01 & 2 & 0.02 & 1 & 0.01 & 1 & 0.010 & 5 & 0.05 \\
\hline Nov. & 3 & 0.03 & 3 & 0.03 & 4 & 0.04 & 2 & 0.020 & 12 & 0.12 \\
\hline Autumn & 9 & 0.03 & 5 & 0.017 & 7 & 0.023 & 5 & 0.050 & 26 & 0.087 \\
\hline G. total & 41 & 0.034 & 17 & 0.014 & 33 & 0.027 & 34 & 0.028 & 125 & 0.104 \\
\hline
\end{tabular}


Table (1): Seasonal distribution of rodent species in the field crops and woodlands, at Al-Azhar University Exptl. Farm, Assiut (2003-2004).

\begin{tabular}{|c|c|c|c|c|c|c|c|c|c|c|c|c|c|c|c|c|}
\hline \multirow{2}{*}{ Site } & \multirow{2}{*}{ Season } & \multicolumn{3}{|c|}{$\begin{array}{c}\text { Arvicanthus } \\
\text { niloticus }\end{array}$} & \multicolumn{3}{|c|}{$\begin{array}{c}\text { Rattus rattus } \\
\text { Frugivorus }\end{array}$} & \multicolumn{3}{|c|}{$\begin{array}{l}\text { Rattus rattus } \\
\text { Alexandrinus }\end{array}$} & \multicolumn{3}{|c|}{ Mus musculus } & \multicolumn{3}{|c|}{ Total rodent } \\
\hline & & \begin{tabular}{|l|} 
No. \\
rat
\end{tabular} & $\%$ & $\begin{array}{c}\text { Trap } \\
\text { index }\end{array}$ & $\begin{array}{c}\text { No. } \\
\text { rat }\end{array}$ & $\%$ & $\begin{array}{c}\text { Trap } \\
\text { index }\end{array}$ & $\begin{array}{l}\text { No. } \\
\text { rat }\end{array}$ & $\%$ & $\begin{array}{c}\text { Trap } \\
\text { index }\end{array}$ & $\begin{array}{l}\text { No. } \\
\text { mice }\end{array}$ & $\%$ & $\begin{array}{c}\text { Trap } \\
\text { index }\end{array}$ & $\begin{array}{l}\text { No. } \\
\text { rodent }\end{array}$ & $\%$ & $\begin{array}{l}\text { Trap } \\
\text { index }\end{array}$ \\
\hline \multirow{5}{*}{ Field crops } & Winter & 42 & 28.6 & 0.140 & 11 & 84.6 & 0.036 & 0 & 0 & 0 & 28 & 40.0 & 0.093 & 81 & 34.8 & 0.270 \\
\hline & Spring & 25 & 17.0 & 0.083 & 1 & 7.7 & 0.003 & 0 & 0 & 0 & 21 & 30.0 & 0.070 & 47 & 20.2 & .156 \\
\hline & Summer & 54 & 36.7 & 0.180 & 1 & 7.7 & 0.003 & 1 & 33.3 & 0.003 & 15 & 21.4 & 0.050 & 71 & 30.4 & .237 \\
\hline & Autumn & 26 & 17.7 & 0.086 & 0 & 0 & 0 & 2 & 66.7 & 0.007 & 6 & 8.6 & 0.020 & 34 & 14.6 & .113 \\
\hline & Total & 147 & 63.09 & 0.122 & 13 & 5.58 & 0.011 & 3 & 1.29 & 0.003 & 70 & 30.04 & 0.058 & 233 & 100 & .194 \\
\hline \multirow{5}{*}{ Woodlands } & Winter & 6 & 7.4 & 0.020 & 0 & 0 & 0 & 13 & 25.5 & 0.043 & 3 & 4.6 & 0.010 & 22 & 10.1 & 0.073 \\
\hline & Spring & 45 & 55.5 & 0.150 & 8 & 40.0 & 0.027 & 20 & 39.2 & 0.067 & 32 & 49.2 & 0.107 & 105 & 48.4 & 0.350 \\
\hline & Summer & 14 & 17.3 & 0.047 & 9 & 45.0 & 0.030 & 7 & 13.7 & 0.023 & 24 & 36.9 & 0.080 & 54 & 24.9 & 0.180 \\
\hline & Autumn & 16 & 19.8 & 0.053 & 3 & 15.0 & 0.010 & 11 & 21.6 & 0.037 & 6 & 9.3 & 0.020 & 36 & 16.6 & 0.120 \\
\hline & Total & 81 & 37.33 & 0.068 & 20 & 9.22 & 0.017 & 51 & 23.5 & 0.043 & 65 & 29.95 & 0.054 & 217 & 100 & 0.181 \\
\hline \multirow{5}{*}{$\begin{array}{l}\text { Field } \\
\text { and } \\
\text { Woodlands }\end{array}$} & Winter & 48 & 21.0 & 0.080 & 11 & 33.3 & 0.018 & 13 & 24.1 & 0.022 & 31 & 23.0 & 0.052 & 103 & 22.9 & 0.172 \\
\hline & Spring & 70 & 30.7 & 0.117 & 9 & 27.3 & 0.015 & 20 & 37.0 & 0.033 & 53 & 39.2 & 0.088 & 152 & 33.8 & 0.253 \\
\hline & Summer & 68 & 29.8 & 0.113 & 10 & 30.3 & 0.017 & 8 & 14.8 & 0.013 & 39 & 28.9 & 0.065 & 125 & 27.8 & 0.208 \\
\hline & Autumn & 42 & 18.5 & 0.070 & 3 & 9.1 & 0.005 & 13 & 24.1 & 0.022 & 12 & 8.9 & 0.020 & 70 & 15.5 & 0.117 \\
\hline & Total & 228 & 50.67 & 0.095 & 33 & 7.33 & 0.014 & 54 & 12.0 & 0.023 & 135 & 30.0 & 0.056 & 450 & 100 & 0.188 \\
\hline
\end{tabular}


Table (2): Seasonal distribution of rodent species in the field crops and woodlands, at Al-Azhar University Exptl. Farm, Assiut (2004-2005).

\begin{tabular}{|c|c|c|c|c|c|c|c|c|c|c|c|c|c|c|c|c|}
\hline \multirow{2}{*}{ Site } & \multirow{2}{*}{ Season } & \multicolumn{3}{|c|}{$\begin{array}{c}\text { Arvicanthus } \\
\text { niloticus }\end{array}$} & \multicolumn{3}{|c|}{$\begin{array}{l}\text { Rattus rattus } \\
\text { Frugivorus }\end{array}$} & \multicolumn{3}{|c|}{$\begin{array}{l}\text { Rattus rattus } \\
\text { Alexandrinus }\end{array}$} & \multicolumn{3}{|c|}{ Mus musculus } & \multicolumn{3}{|c|}{ Total rodent } \\
\hline & & \begin{tabular}{|c|}
$\begin{array}{c}\text { No. } \\
\text { rat }\end{array}$ \\
\end{tabular} & $\%$ & $\begin{array}{c}\text { Trap } \\
\text { index }\end{array}$ & $\begin{array}{l}\text { No. } \\
\text { rat }\end{array}$ & $\%$ & $\begin{array}{c}\text { Trap } \\
\text { index }\end{array}$ & $\begin{array}{l}\text { No. } \\
\text { rat }\end{array}$ & $\%$ & $\begin{array}{c}\text { Trap } \\
\text { index }\end{array}$ & $\begin{array}{l}\text { No. } \\
\text { mice }\end{array}$ & $\%$ & \begin{tabular}{|c|} 
Trap \\
index
\end{tabular} & $\begin{array}{c}\text { No. } \\
\text { rodent }\end{array}$ & $\%$ & \begin{tabular}{|c|} 
Trap \\
index
\end{tabular} \\
\hline \multirow{5}{*}{ Field crops } & Winter & 16 & 20.0 & 0.053 & 4 & 100 & 0.013 & 1 & 16.7 & 0.003 & 1 & 4.8 & 0.003 & 22 & 19.8 & 0.073 \\
\hline & Spring & 17 & 21.2 & 0.057 & 0 & 0 & 0 & 1 & 16.7 & 0.003 & 3 & 14.3 & 0.010 & 21 & 18.9 & 0.070 \\
\hline & Summer & 29 & 36.3 & 0.097 & 0 & 0 & 0 & 0 & 0 & 0 & 8 & 38.1 & 0.027 & 37 & 33.3 & 0.123 \\
\hline & Autumn & 18 & 22.5 & 0.060 & 0 & 0 & 0 & 4 & 66.6 & 0.013 & 9 & 42.8 & 0.030 & 31 & 28.0 & 0.103 \\
\hline & Total & 80 & 72.07 & 0.067 & 4 & 3.6 & 0.003 & 6 & 5.41 & 0.005 & 21 & 18.92 & 0.018 & 111 & 100 & 0.092 \\
\hline \multirow{5}{*}{ Woodlands } & Winter & 6 & 14.6 & 0.020 & 0 & 0 & 0 & 13 & 39.4 & 0.043 & 3 & 8.8 & 0.010 & 22 & 17.6 & 0.073 \\
\hline & Spring & 16 & 39.0 & 0.053 & 3 & 17.6 & 0.010 & 4 & 12.1 & 0.013 & 10 & 29.4 & 0.033 & 33 & 26.4 & 0.110 \\
\hline & Summer & 10 & 24.4 & 0.033 & 9 & 53.0 & 0.030 & 9 & 27.3 & 0.030 & 16 & 47.0 & 0.053 & 44 & 35.2 & 0.147 \\
\hline & Autumn & 9 & 22.0 & 0.030 & 5 & 29.4 & 0.017 & 7 & 21.2 & 0.023 & 5 & 14.8 & 0.017 & 26 & 20.8 & 0.087 \\
\hline & Total & 41 & 32.8 & 0034 & 17 & 13.6 & 0.014 & 33 & 26.4 & 0.027 & 34 & 27.2 & 0.028 & 125 & 100 & 0.104 \\
\hline Field & sWinter & 22 & 18.2 & 0.036 & 4 & 19.0 & 0.007 & 14 & 35.9 & 0.023 & 4 & 7.3 & 0.007 & 44 & 18.6 & 0.073 \\
\hline
\end{tabular}




\section{Abdel-gawad, K.H. et al.}

\begin{tabular}{|c|c|c|c|c|c|c|c|c|c|c|c|c|c|c|c|c|}
\hline \multirow{4}{*}{$\begin{array}{l}\text { and } \\
\text { Woodlands }\end{array}$} & Spring & 33 & 27.3 & 0.055 & 3 & 14.3 & 0.005 & 5 & 12.8 & 0.008 & 13 & 23.6 & 0.022 & 54 & 22.9 & 0.090 \\
\hline & Summer & 39 & 32.2 & 0.065 & 9 & 42.9 & 0.015 & 9 & 23.1 & 0.015 & 24 & 43.6 & 0.040 & 81 & 34.3 & 0.135 \\
\hline & Autumn & 27 & 22.3 & 0.045 & 5 & 23.8 & 0.008 & 11 & 28.2 & 0.018 & 14 & 25.5 & 0.023 & 57 & 24.2 & 0.095 \\
\hline & Total & 121 & 51.27 & 0.050 & 21 & 8.89 & 0.009 & 39 & 16.53 & 0.016 & 55 & 23.31 & 0.023 & 236 & 100 & 0.098 \\
\hline
\end{tabular}

